\section{REFERÊNCIAS}

Stratta RJ, Taylor RJ, Bynon S, Lowell JA, et al. Surgical treatment of diabetes mellitus with pancreas transplantation. Annals of Surgery. 1994; 220(6): 809-17.

2. Sollinger HW, Odorico JS, Knechtle SJ, D'Alessandro AM, et al. Experience with 500 simultaneous pancreas-kidney transplants. Annals of Surgery. 1998; 228(3): 284-96.

3. Sutherland DER, Gruessner RWG, Najarian JS, Gruessner AC. Solitary pancreas transplant: a new era. Transplantation Proceedings. 1998; 30:280-1.

4. Bilous RW, Mauer M, Sutherland DER, Najarian JS, et al. The effects of pancreas transplantation on the glomerular structure of renal allografts in patients with insulindependent diabetes. The New England Journal of Medicine. 1989; 321(2):80-5.

5. Fioretto P, Mauer SM, Bilous RW, Goetz FC, et al. Effects of pancreas transplantation on glomerular structure in insulin-dependent diabetic patients with their own kidneys. The Lancet. 1993; 342: 1193-6.

6. Fioretto P, Steffes MW, Sutherland DER, Goetz FC, et al. Reversal of lesions of diabetic nephropathy after pancreas transplantation. The New England Journal of Medicine. 1998; 339(2): 69-75.
7. Kennedy WR, Navarro X, Goetz FC, Sutherland DER, et al. Effects of pancreatic transplantation on diabetic neuropathy. The New England Journal of Medicine. 1990; 322(15): 1031-6.

8. Navarro X, Sutherland DER, Kennedy WR. Long-term effects of pancreatic transplantation on diabetic neuropathy. Annals of Neurology. 1997; 42(5): 727-36.

9. Ramsay RC, Goetz FC, Sutherland DER, Mauer SM, et al. Progression of diabetic retinophaty after pancreas transplantation for insulin-dependent diabetes mellitus. The New England Journal of Medicine. 1988; 318(4): 208-13.

10. Stratta RJ. Impact of pancreas transplantation on the complications of diabetes. Current Opinion in Organ Transplantation. 1998; 3(4): 258-73.

11. Sutherland DER, Stratta RJ, Gruessner AC. Pancreas transplant outcome by recipient category: single pancreas versus combined kidney-pancreas. Current Opinion in Organ Transplantation. 1998; 3(4): 231-41.

12. Bartlett ST, Schweitzer EJ, Johnson LB, Kuo PC, et al. Equivalent success of simultaneous pancreas kidney and solitary pancreas transplantation. Annals of Surgery. 1996; 224(4):440-52. 


\title{
TRANSPLANTE DE FÍGADO INTERVIVOS: A VIVÊNCIA DE DOADORES NO PROCESSO DE DOAÇÃO
}

\author{
The living related liver transplant: the experience of donors during the donation process
}

Rosemeire Keiko Hangai', Maria Cristina Komatsu Braga Massarollo²

\begin{abstract}
RESUMO
Objetivo: Desvelar a vivência de doadores no processo de doação para a realização do transplante de fígado intervivos (TxIV) em um hospital público. Método: Para compreender o vivencial dos doadores optou-se por realizar uma pesquisa com abordagem qualitativa, utilizando a vertente fenomenológica, segundo a modalidade estrutura do fenômeno situado. Para a realização da pesquisa foram entrevistados sete doadores, que já haviam concluído o processo de preparação e estavam aguardando a realização do procedimento. Resultado: A análise dos discursos evidenciou os seguintes temas: "a busca de uma solução para a doença do filho", "o conhecimento da possibilidade de realização do TxIV", "a decisão de doar", "o preparo para a realização do TxIV", "a espera para a realização do TxIV" e "a importância da família no processo". Conclusão: A essência do fenômeno "a vivência de doadores no processo de doação para a realização do transplante de fígado intervivos" foi desvelada como sendo uma situação de buscas e dificuldades para encontrar a solução para a doença do filho, de certeza na decisão de doar parte do fígado para salvar o filho, de ansiedade no período de espera, de medo de perder o filho antes da realização do procedimento e de esperança no sucesso do transplante.
\end{abstract}

Palavras-chave: Transplante de fígado, Doadores vivos, Vivência.

\footnotetext{
Trabalho realizado na unidade Instituto da Criança "Pedro Alcântara" - Hospital das Clínicas - Faculdade de Medicina da USP.

1. Enfermeira, Mestre em Enfermagem pela Escola de Enfermagem da USP:

2. Professora Doutora da Escola de Enfermagem da USP.

Endereço para correspondência: Rosemeire Keiko Hangai - Rua João Hoffmann, 168 VI Aliança - Osasco - São Paulo - SP - CEP 06060-130

Fone: (11) 3691-0577 - e-mail: rosemeirek@yahoo.com.br

Recebido em: 30/06/2003 Aceito em: 12/01/2005
}

\section{INTRODUÇÃO}

O transplante de fígado é uma modalidade terapêutica que possibilita a reversão de lesões orgânicas terminais, representando para muitos a possibilidade de viver. ${ }^{1}$ Com o avanço das técnicas cirúrgicas e de preservação de órgãos, além do desenvolvimento de drogas imunossupressoras, no início dos anos 80 , o número de transplantes aumentou nos EUA e Europa, com melhores resultados. Entretanto, houve, também, o aumento do número de pacientes portadores de doenças hepáticas agudas e crônicas, com indicação de transplante de fígado, permanecendo a insuficiência de enxertos disponíveis, e, até, aumentando a desproporção existente entre a oferta e a demanda de órgãos para serem transplantados. Essa carência parece ainda maior para pacientes pediátricos. ${ }^{2,3}$

$\mathrm{Na}$ tentativa de solucionar a escassez de doadores pediátricos e diminuir a mortalidade dos pacientes em lista de espera, começaram a surgir algumas alternativas e, dentre elas, o transplante de fígado intervivos (TxIV). Esse procedimento foi realizado pela primeira vez no Brasil, em 1989. ${ }^{6}$ Para a realização desse procedimento cirúrgico são utilizados enxertos provenientes de doadores vivos, sendo, normalmente, retirada parte do lobo esquerdo do fígado de um doador adulto, para ser implantado em um receptor pediátrico. ${ }^{4,5,6}$ Após dez anos de experiência com transplante hepático pediátrico com doador cadáver, iniciou-se, em 1998, o programa de transplante hepático com doador vivo na instituição local do estudo. 
O acompanhamento dos pais doadores, no processo de realização do TXIV, possibilitou observar a manifestação de medo da cirurgia e da evolução, a ansiedade de ver o filho após o transplante, a incerteza do sucesso do procedimento, a alegria do sucesso, o sofrimento e a frustração quando havia a necessidade de um retransplante e, quando da perda do filho, o desespero, a raiva e a tristeza, mostrando o sofrimento do doador e a complexidade da situação. A observação dessas situações despertou indagações referentes à vivência do doador para a realização do transplante de fígado intervivos.

\section{OBJETIVO}

Desvelar a vivência de doadores no processo de doação para a realização do transplante de fígado intervivos em um hospital público.

\section{MÉTODO}

Para o alcance do objetivo proposto optou-se por uma abordagem qualitativa, utilizando a vertente fenomenológica, modalidade estrutura do fenômeno situado. ${ }^{7}$ A adoção do método fenomenológico na pesquisa visou captar o fenômeno, possibilitando sua compreensão. A região de inquérito, no presente estudo, foi a situação de vivenciar o processo de doação de parte do fígado para a realização de transplante no Programa de Transplante Hepático Intervivos, de um hospital público de ensino, do município de São Paulo, compreendendo a doação como um processo que engloba as seguintes fases: a indicação de transplante para a criança ou adolescente, a manifestação do desejo de ser doador, a realização dos exames protocolares, a confirmação da possibilidade de doar parte do órgão, a espera para a realização do procedimento, o transplante propriamente dito e o período pós-operatório.

Os sujeitos que vivenciavam o fenômeno e que participaram do estudo eram doadores* que já haviam concluído o processo de preparação e estavam aguardando a realização do procedimento. Os discursos foram coletados após a autorização da instituição, a aprovação do Comitê de Ética em Pesquisa, o esclarecimento dos sujeitos e a assinatura do Termo Consentimento Livre e Esclarecido, utilizando as seguintes questões norteadoras: "Como você ficou sabendo da possibilidade de realização do transplante de fígado intervivos?", "Como foi para você tomar a decisão de ser doador?" e "Como foi o período de preparo e está sendo o de espera para a realização do transplante?". Conforme o método adotado, o número de sujeitos participantes da pesquisa não foi definido a priori, mas, sim, com a ocorrência da repetitividade dos discursos. Dessa forma, participaram do estudo sete potenciais doadores, sendo que, desses, cinco eram mães e dois eram pais.

Para a análise das entrevistas, seguiram-se os momentos metodológicos do fenômeno situado: o sentido do todo, a discriminação das unidades de significado, a transformação das expressões do sujeito em linguagem do pesquisador e a síntese das unidades de significado transformadas em proposições, possibilitando, assim, o desvelamento da estrutura do fenomeno situado.

*Neste estudo foi utilizada para os sujeitos da pesquisa a denominação de doadores e potenciais doadores, indistintamente.

\section{RESULTADO}

$\mathrm{O}$ fenômeno desvelado nesta pesquisa foi a vivência do processo de doação de parte do fígado para a realização de transplante no Programa de Transplante Hepático Intervivos, de um hospital público de ensino, do município de São Paulo".
Experienciar esse processo revelou que há dificuldade na busca de um tratamento para a doença, relativa ao longo tempo de procura, há desinformação sobre a doença e há falta de vagas para a internação. Essas dificuldades geram um sentimento de incapacidade e desespero nos pais, pela possibilidade de piora do quadro clínico da criança, levando, até, a uma situação irreversível.

Quanto ao conhecimento sobre a possibilidade de realização do procedimento, foi revelado que ele é obtido através de médicos e enfermeira da equipe de transplante da instituição, do manual do programa de transplantes, de livros e, também, através de outros pais que já passaram, ou que estão passando pelo processo de doação. O contato com os pais das crianças, que já foram submetidos ao TxIV possibilita a troca de informações e de experiências, fazendo com que os potenciais doadores adquiram confiança e coragem para a realização do procedimento.

O TxIV surge como uma opção terapêutica que tem como vantagem ser mais rápido do que o transplante com doador cadáver. $\mathrm{O}$ longo tempo de espera para a realização do transplante de fígado pediátrico com doador cadáver faz com que os pais optem pelo TxIV. Além disso, é considerado um procedimento novo, com muita probabilidade de sucesso.

Quanto à decisão de doar, os pais, mesmo conhecendo os riscos relacionados ao procedimento, manifestam o desejo de doação de parte do fígado para o filho. A vontade de salvar a vida do filho é tão intensa que desconsideram os riscos a que estarão sendo submetidos na realização do transplante, não tendo dúvida quanto à decisão tomada. A batalha para encontrar uma solução é tão árdua que, quando a encontram, aceitam-na com facilidade, não tendo dificuldades na tomada de decisão quanto à escolha do tratamento e nem quanto a serem doadores. O desejo dos pais é salvar a vida do filho e amenizar o seu sofrimento e acreditam que, com a ajuda de Deus, conseguirão fazê-lo. Para as mães, o fato de "serem mães" lhes dá a convicção de doar parte do fígado para o filho, relacionando essa decisão com o renascimento do filho, com o "dar a vida novamente". Quando ocorre sofrimento e desespero na mãe, decorrentes do impacto gerado pela sua indicação para ser a potencial doadora, esses são minimizados pela compreensão de que o transplante de fígado é a única possibilidade terapêutica para a sobrevivência do filho.

Em relação ao preparo para a realização do procedimento, os exames preparatórios, feitos pelo potencial doador, são doloridos. A dor e o desconforto provocados pela realização dos exames, em nenhum momento, são motivos que levam os pais a pensar em desistir da doação. Durante a realização dos exames, morar longe do centro transplantador traz dificuldades para os doadores. A demora na realização desses exames provoca um sentimento de ansiedade, o que concorre para aumentar, ainda mais, o sofrimento dos pais que, durante a realização dos exames, ficam ansiosos para saber se poderão ser doadores, ou não, e, nesse momento, o pensamento é positivo, acreditando que os resultados serão favoráveis.

Quanto à espera, a realização do transplante é aguardada com muita expectativa, pois a demora é motivo de preocupação para os pais. Eles temem que algo aconteça com o filho e que a não realização do transplante possa significar a "morte" do mesmo.

O desejo dos pais é que após a realização do procedimento a criança tenha uma nova vida, livre de restrições e sofrimentos. Quanto à família, ficou evidente a sua importância nesse processo, pois os pais necessitam de apoio e segurança das pessoas que são significativas, na difícil situação de espera pela realização do transplante de fígado intervivos. 


\section{CONCLUSÃO}

A essência do fenômeno "a vivência de doadores no processo de doação para a realização do transplante de fígado intervivos" foi desvelada como sendo uma situação de buscas e dificuldades para encontrar a solução para a doença do filho, de certeza na decisão de doar parte do fígado para salvar o filho, de ansiedade no período de espera, de medo de perder o filho antes da realização do procedimento e de esperança no sucesso do transplante.

\section{ABSTRACT}

Objective: The objective of this study was to characterize the experience of donors undergoing the donation process for living related liver transplantation in a public hospital. Methods: A qualitative study was performed according to the modality "structure of the situated phenomenon". Seven potential donors who had already concluded the preparation process and were awaiting the procedure participated in the study. Results: After obtaining these answers they were analyzed individually, calling attention for the following themes: "The search for a solution", "The knowledge of the possibility of doing a Living Related Liver Transplant", "The decision to donate", "The preparation for the liver transplant ", "The time waiting for the Living Related Liver Transplant", "the importance of the family in the process". Conclusion: The essential aspects of the phenomenon were revealed to be those of living through a situation of searching for and difficulties in finding a solution for their child's disease, of certainty in the decision to donate part of their liver to save the child's life, together with anxiety in the waiting period, fear of losing the child before the procedure is performed and hope that the transplant will be successful.

Key words: Liver transplantation, Living donors, Life change events.

\section{REFERÊNCIAS}

Massarollo MCKB. A utilização de doadores vivos para transplante de órgãos. Medicina 1999;14:8-9.

2. Ozawa K. Living related donor liver transplantantion: assessment of graft viability based on the redox theory. Basel: Karger; 1994. p.30-45: Ethical considerations.

3. Chapchap P, Maksoud JG. Doação de órgãos: um problema atual. Rev. Assoc. Med. Brasil 1988;34:190-2.

4. Carone E, Chapchap P, Porta G, Miura I, Pugliesi V, Ayoub A, Averbach M, Cabêdo
MTC, Faria 1S, Tobaldini R, Zumiotti A, Ohnoo P, Dias MBS, Gregory FH, Silva ED, Parise ER, Kondo M. Transplante hepático com doador vivo familiar.

J Pediatr 1998; 74:99-106.

5. Maksoud JG. Transplante de fígado infantil. Médicos HC-FMUSP 1999; 2:76-86.

6. Ozawa K. Living related donor liver transplantantion: assessment of graft viability based on the redox theory. Basel: Karger; 1994. p.1-11: Historical Background.

7. Martins J, Bicudo MAV. A pesquisa qualitativa em psicologia: fundamentos e recursos básicos. São Paulo: Moraes/ EDUC; 1989. 